\title{
Teaching about photodetection noise sources in the laboratory
}

Lionel Jacubowiez, Jean-Francois Roch, Jean-Philippe Poizat, Philippe Grangier

Lionel Jacubowiez, Jean-Francois Roch, Jean-Philippe Poizat, Philippe Grangier, "Teaching about photodetection noise sources in the laboratory," Proc. SPIE 3190, Fifth International Topical Meeting on Education and Training in Optics, (8 December 1997); doi: 10.1117/12.294378

Event: Fifth International Topical Meeting on Education and Training in Optics, 1997, Delft, Netherlands 


\title{
Teaching photodetection noise sources in laboratory
}

Lionel Jacubowiez, Jean-François Roch, Jean-Philippe Poizat and Philippe Grangier Institut d'Optique, Ecole Superieure d'Optique and CNRS associated

Centre Universitaire, BP 147, Orsay 91403 France

\begin{abstract}
We describe simple experiments that allow students to observe, identify, understand and measure different noise sources always present in photodetection systems : amplifier noise, thermal resistance noise (Johnson noise), and photon noise (shot-noise).

With a suitable low noise amplifier and a commercial photodiode, students can verify the dependence of photon noise versus light level.

This photon shot-noise is directly related to the quantum " nature " of light and it has long been considered as a fundamental limitation of the optical photodetection systems. It is sometimes mistakenly described as due to the detector itself.

We show that it is possible, with fairly affordable laboratory teaching equipment, to measure a photocurrent with a noise power below the shot-noise level using a suitable light source. More precisely, using a photodiode and a high-quantum-efficiency light-emitting diode driven by a constant current source, we can observe a reduction of the photon noise power of about $0.8 \mathrm{~dB}$ below the shot-noise level.
\end{abstract}

Keywords: Noise sources, photodetection, shot-noise, sub-Poissonian statistics 


\section{1- Amplification noise}

\subsection{Experiment equipment:}

We will use a low-noise and high gain amplifier to measure the amplifier noise and study the thermal noise of a resistance.

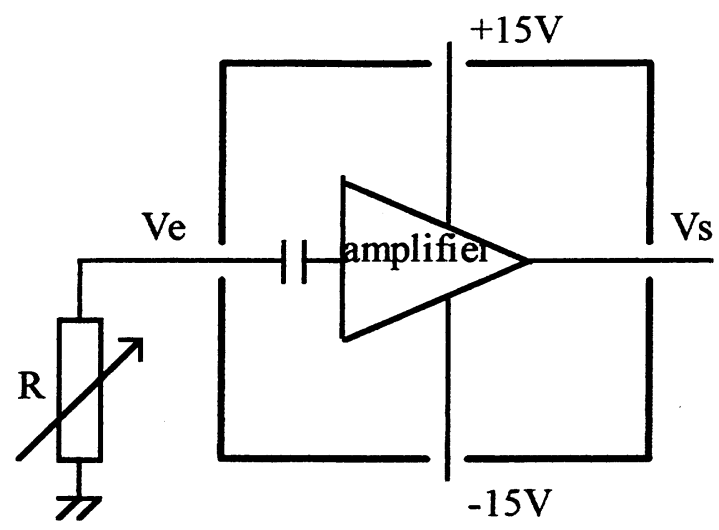

Amplifier : thermal noise

Fig. 1: circuit description at the end of this paper

This is a 2 stages very low noise amplifier with a gain of about 250 and a bandwidth of $50 \mathrm{kHz}$ to 1.6 Mhz. The first stage is made with an integrated amplifier (AH 0013) whose voltage noise is $2 \mathrm{nV} / \sqrt{\mathrm{Hz}}$, according to the data sheet. The contribution of the current noise, $10 \mathrm{fA} / \sqrt{\mathrm{Hz}}$, is always negligible. The second stage is a more usual amplifier, OP37 (Burr and Brown).

Of course, the circuit must be done carefully, placed in a metal box, connected by coaxial cables. Furthermore, storage battery operation may be useful to avoid noise caused by the power supply.

\subsection{Noise amplifier measurement:}

When the input is grounded, voltage amplifier output noise can be observed with an ordinary oscilloscope, and measurements can be done either with an oscilloscope or a spectrum analyzer. Using both apparatus is interesting because an oscilloscope allows the students to get familiar with the temporal aspect of noise (temporal fluctuations are often a Gaussian distribution), and a spectrum analyzer helps to get familiar with the frequency aspect of noise (is it a white noise?).

\section{2.a : Noise measurement with an oscilloscope :}

With an ordinary oscilloscope one can estimate the peak to peak output voltage noise of the amplifier. Assuming this noise is a Gaussian noise, which means that the temporal statistical fluctuation of the output voltage is a Gaussian curve centered on zero, we know that $: V_{R M S, \text { out }} \approx \frac{V_{\text {peak to peak }}}{6}$.

A digital oscilloscope (HP57604B for example) allows us to measure directly $V_{R M S}$ and $V_{\text {peak to peak }}$, and thus, to verify this relation.

This root mean square output voltage is related to the input voltage noise, $v_{N, i n}$, of the amplifier :

$$
V_{R M S, \text { out }}^{2}=\int_{0}^{\infty} \operatorname{Gain}(f)^{2} v_{N, \text { in }}^{2} d f
$$

In this calculation we assume that the amplifier input noise is white (which is true inside the bandwidth of our amplifier) and that all the spectral contributions of the amplifier noise are statistically independent. Futhermore if we suppose that the amplifier is a first order low pass filter, the amplifier equivalent noise bandwidth, $E N B W$,

is: $\sqrt{\frac{\pi}{2} f_{-3 d B}}$. In this case the amplifier voltage input noise is: $v_{N, i n}=\frac{\mathrm{V}_{\mathrm{RMS}} \text {, out }}{\text { Gain } \sqrt{\frac{\pi}{2} f_{-3 d B}}}$ in $\mathrm{V} / \sqrt{\mathrm{Hz}}$. 
1.2.b : Noise measurement with a spectrum analyzer :

With a spectrum analyzer (Tektronix 2712 for example), one can observe the amplifier noise output power versus frequency (fig. 3). Furthermore, a spectrum analyzer allows us also to measure directly the spectral density power of noise at a given frequency. This power is given in $\mathrm{dBm}$ for an equivalent noise bandwidth of $1 \mathrm{~Hz}: 10 \log \left(\frac{P(W / H z)}{10^{-3}}\right)$. Assuming the input resistance of the spectrum analyzer is $50 \Omega$ :

$$
P(\text { in } W / H z)=\frac{V^{2}{ }_{N, \text { out }}(\text { in } V / \sqrt{H z})}{50}
$$

Thus the amplifier input voltage noise is given by:

$$
V_{N, \text { in }}(\text { in } V / \sqrt{H z})=\frac{1}{\text { Gain }}\left[50 \cdot 10^{-3} \cdot 10^{\left(\frac{P(\text { in a ABm for } 1 \mathrm{~Hz} E N B W)}{10}\right)}\right]^{\frac{1}{2}}
$$

The gain should be measured separately and carefully, using a very small voltage signal (few $\mathrm{mV}$ ) at the same frequency.

Using this noise measurement method, we measure for our amplifier at $40 \mathrm{kHz}: V_{N, \text { in }}=2 \mathrm{nV} / \sqrt{\mathrm{Hz}} \pm 0.2$ which agrees with the data sheet value.

\section{2- Thermal resistance noise}

With this amplifier it is easy to study the thermal noise (Johnson noise) of a resistance. The voltage noise source is given by the Johnson-Nyquist formula :

$$
\begin{gathered}
v_{N, R}=\sqrt{4 k T R} \text { in } \mathrm{V} / \sqrt{\mathrm{Hz}} \\
\text { where } k \text { is the Boltzmann constant: } \quad k=1.3810^{-23} \mathrm{~J} . \mathrm{K}^{-1} .
\end{gathered}
$$

\subsection{Influence of the resistance:}

We simply measure the amplifier voltage output noise with different resistances placed at the amplifier input. Figure 2 shows $v_{n R}$ versus $\sqrt{R}$ measured at $40 \mathrm{kHz}$.

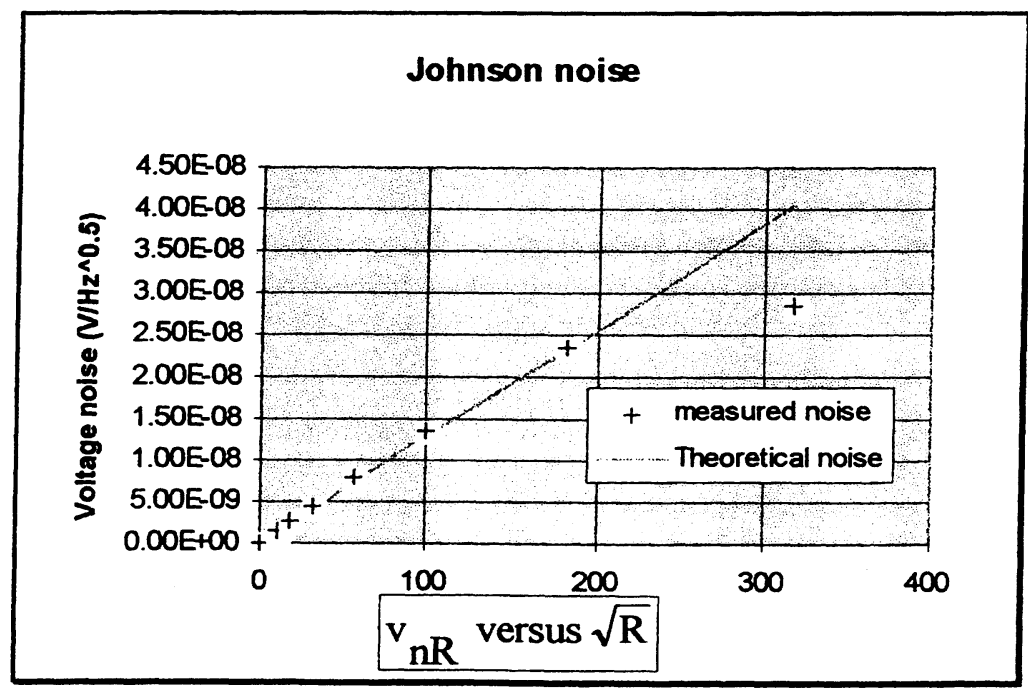

Fig. 2 : Experimental and theoretical Jonhson noise $v_{n R}$ versus $\sqrt{R}$ 


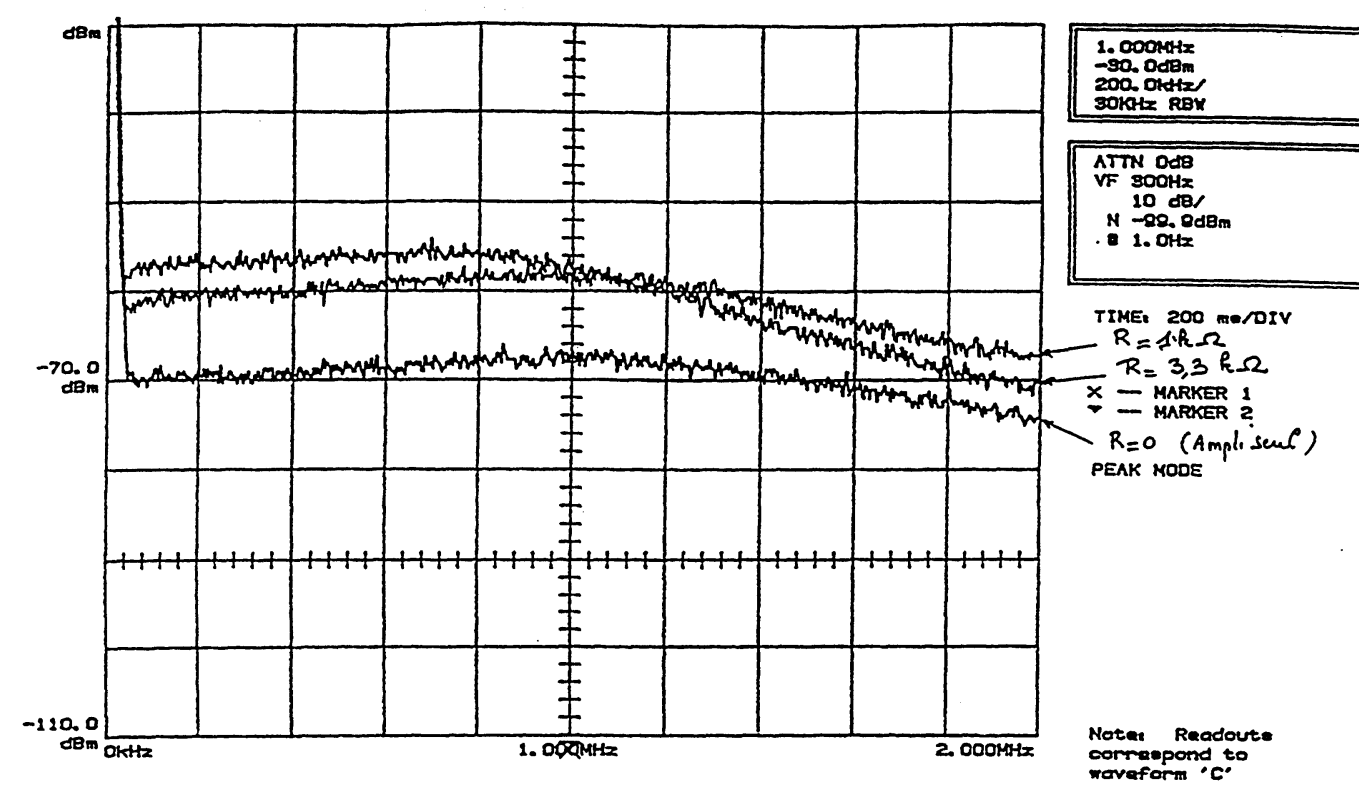

fig. 3

Amplifier output noise power for $0,1 \mathrm{k} \Omega$ et $3.3 \mathrm{k} \Omega$ input resistance

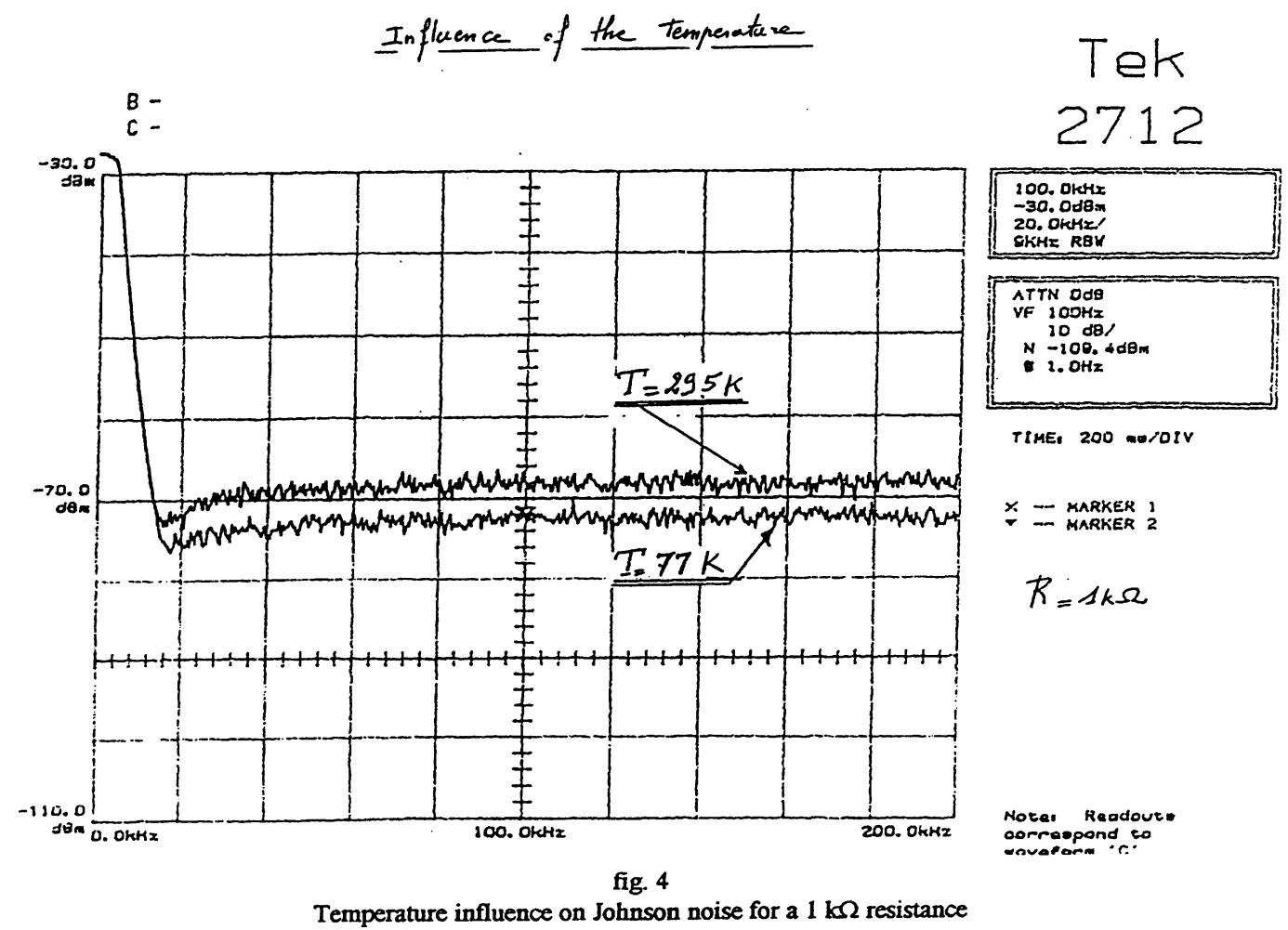




\section{Remarks:}

1- Measurements for resistance above $40 \mathrm{k} \Omega$ are obviously false. This is due to a $40 \mathrm{pF}$ stray capacitance (amplifier input capacitance and connection cable capacitance) which makes a low pass filter whose cut off frequency depends on the resistance measured (for $\mathrm{R}=100 \mathrm{k} \Omega, f_{c}=40 \mathrm{kHz}$ ).

2- Thermal noise is given by $: v_{N, R}=\sqrt{\frac{v_{R M S, \text { out }}^{2}}{\text { Gain }}-v_{N, \text { in }}^{2}}$ because amplifier noise and resistance thermal noise are statistically independent.

3- We could expect to use this experiment to estimate Boltzmann's constant $k$, but generally the spectrum analyzer is not calibrated precisely enough. On the other hand, it is possible to use these results to calibrate the spectrum analyzer for noise measurements. Then, photon noise measurements in the next part will fit the theory and give the opportunity to measure the charge of an electron.

\subsection{Influence of the temperature:}

We place a $1 \mathrm{k} \Omega$ resistance in a small metal box connected to the amplifier input, and we put it in liquid nitrogen. Compare to the noise at room temperature, we measure a thermal noise divided by 1.96 (fig. 4). This simple experiment shows the principle of a noise thermometer measuring the absolute temperature (in Kelvin).

\section{3- Photon noise}

\subsection{Shot-noise theory:}

The photon noise is related to the statistical fluctuation of the photons collected by the photodetector. The photon counting statistics is known to be Poissonian : if the dectector surface receives $\boldsymbol{N}$ photons in average during an integration time $\tau$, the standart deviation of the number of photons received is $\sqrt{N}$. The photoelectrons created in the detector obey the same Poissonian statistics and this explains the shot-noise on the photocurrent. The variance of the fluctuation of the photocurrent, $I_{p h}$, is given by Schottky formula:

$$
\left\langle\Delta i_{p h}^{2}\right\rangle=2 e I_{p h} \Delta f
$$

\subsection{Experiment equipment:}

We study the influence of the light source power level on the shot-noise with this second circuit.

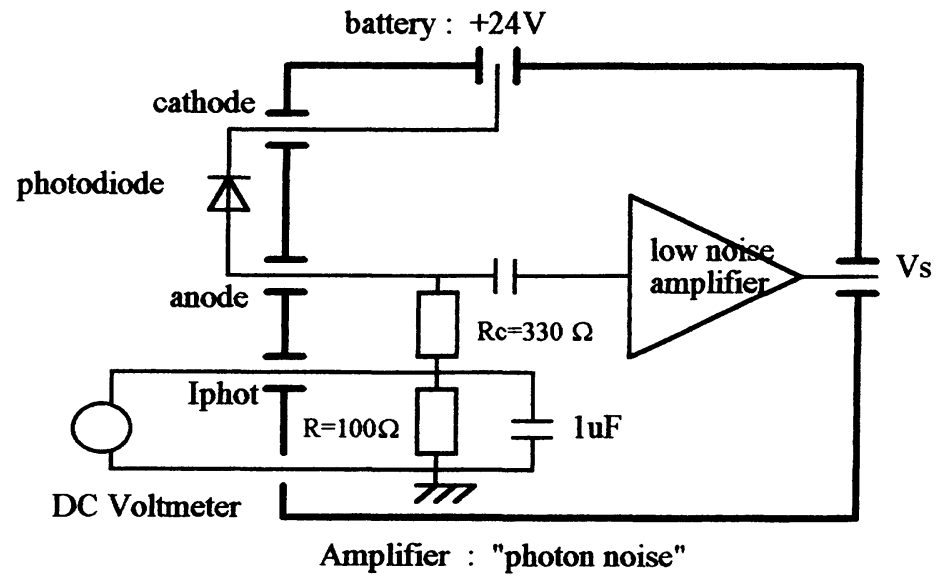

Fig. 5 : Setup of the photon noise experiment 
The low noise amplifier is exactly the same in this circuit. The photodiode is a E.G.G. C30809 whose quantum efficiency is 0.83 at $900 \mathrm{~nm}$.

The shot-noise will be detected as a voltage noise accross the load resitance $R_{c}=330 \Omega$ :

$$
V_{N, \text { phot. }}=R_{c} \sqrt{2 e I_{p h} \Delta f} \text { where } I_{p h} \text { is the mean current in the photodiode. }
$$

This noise is greater than the Johnson noise if : $I_{p h}>\frac{4 k T}{2 e R_{c}} \quad$ (for T=300K, $I_{p h}>0.16 \mathrm{~mA}$ ).

The photocurrent, $I_{p h}$, is measured by a voltmeter connected to $R=100 \Omega$. With an ordinary white lamp, it is easy to obtain a photocurrent from 0 to $12 \mathrm{~mA}$.

\subsection{Influence of the light power :}

With the spectrum analyzer (fig. 7), we compare the amplifier noise output for $I_{p h}=0,2$ and $4 \mathrm{~mA}$. When $I_{p h}=2 \mathrm{~mA}$, shot-noise is already the main source of noise (about $10 \mathrm{~dB}$ above noise power without light). For $I_{p h}=4 \mathrm{~mA}$, noise is $3 \mathrm{~dB}$ above noise for $I_{p h}=2 \mathrm{~mA}$ as predicted by Schottky formula.

We also measure the amplifier voltage noise output at a given frequency (fig. $6, f=40 \mathrm{kHz}$ ) with the spectrum analyser. The photon noise voltage source is given by :

$$
V_{N, \text { phot. }}\left(I_{p h}\right)=\frac{1}{\text { Gain }} \sqrt{V_{R M S, \text { out }}\left(I_{p h}\right)^{2}-V_{R M S, \text { out }}(0)^{2}}
$$

where the gain of the amplifier is measured carefully at the same frequency, and $V_{R M S, \text { out }}(0)$ (output noise for $I_{p h}=0$ ) represents amplifier noise and $R_{c}$ Johnson noise.

We verify that $V_{N, \text { phot. }}\left(I_{p h}\right)$ versus $\sqrt{I_{p h}}$ is a linear function. The slope, $R_{c} \sqrt{2 e}$, gives a good estimate of the charge of an electron.

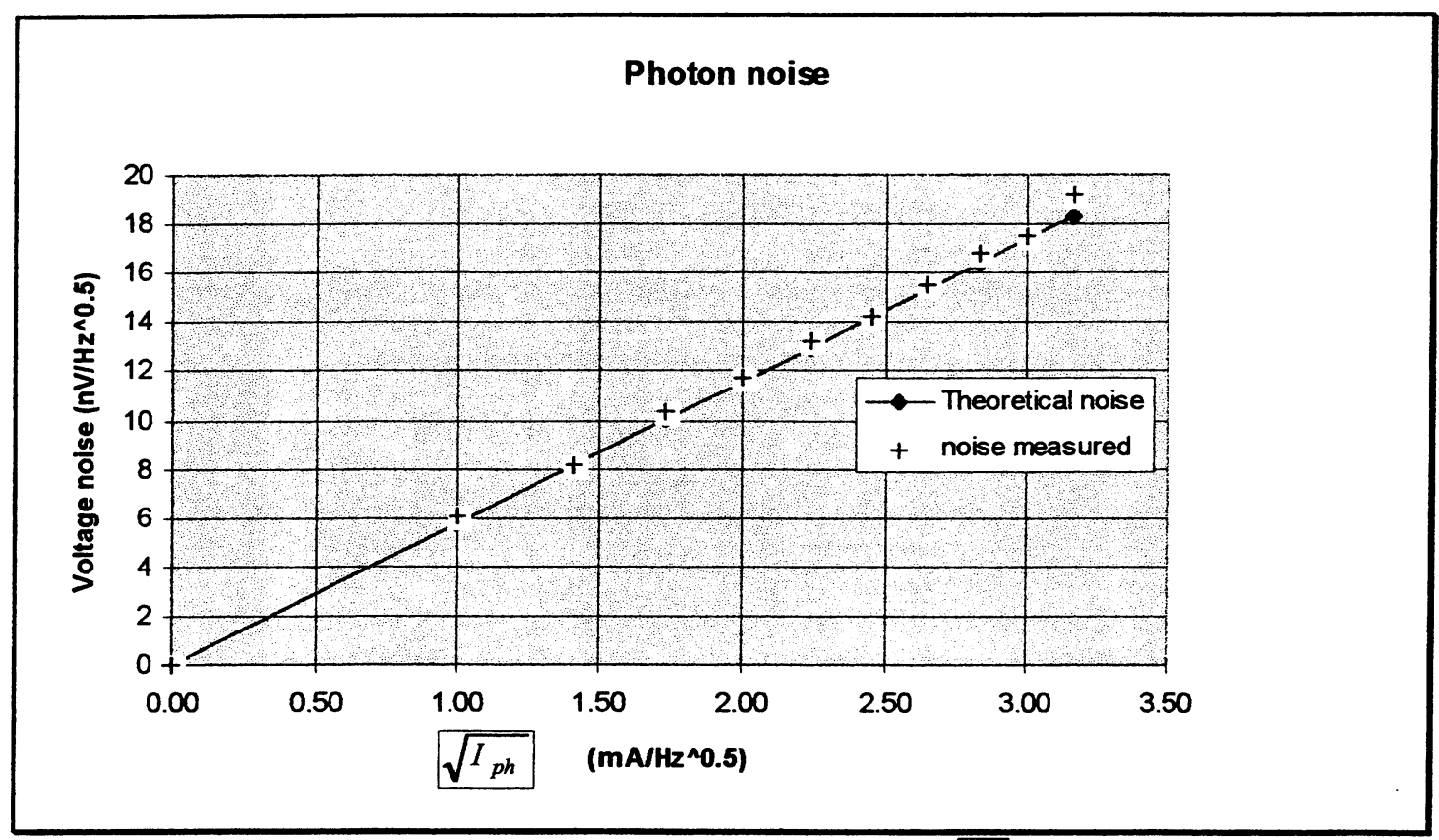

Fig. 6 : Photon noise versus $\sqrt{I_{p h}}$

It is also possible to verify that other ordinary light sources are at the shot-noise limit. Some light sources are well above this limit because of technical noise. For example, figure 8 shows the comparison between a $5 \mathrm{~mW}$ $\mathrm{He}-\mathrm{Ne}$ laser and a white light for the same mean current in the photodiode. The laser He-Ne appears to be very noisy under $1 \mathrm{MHz}$. 

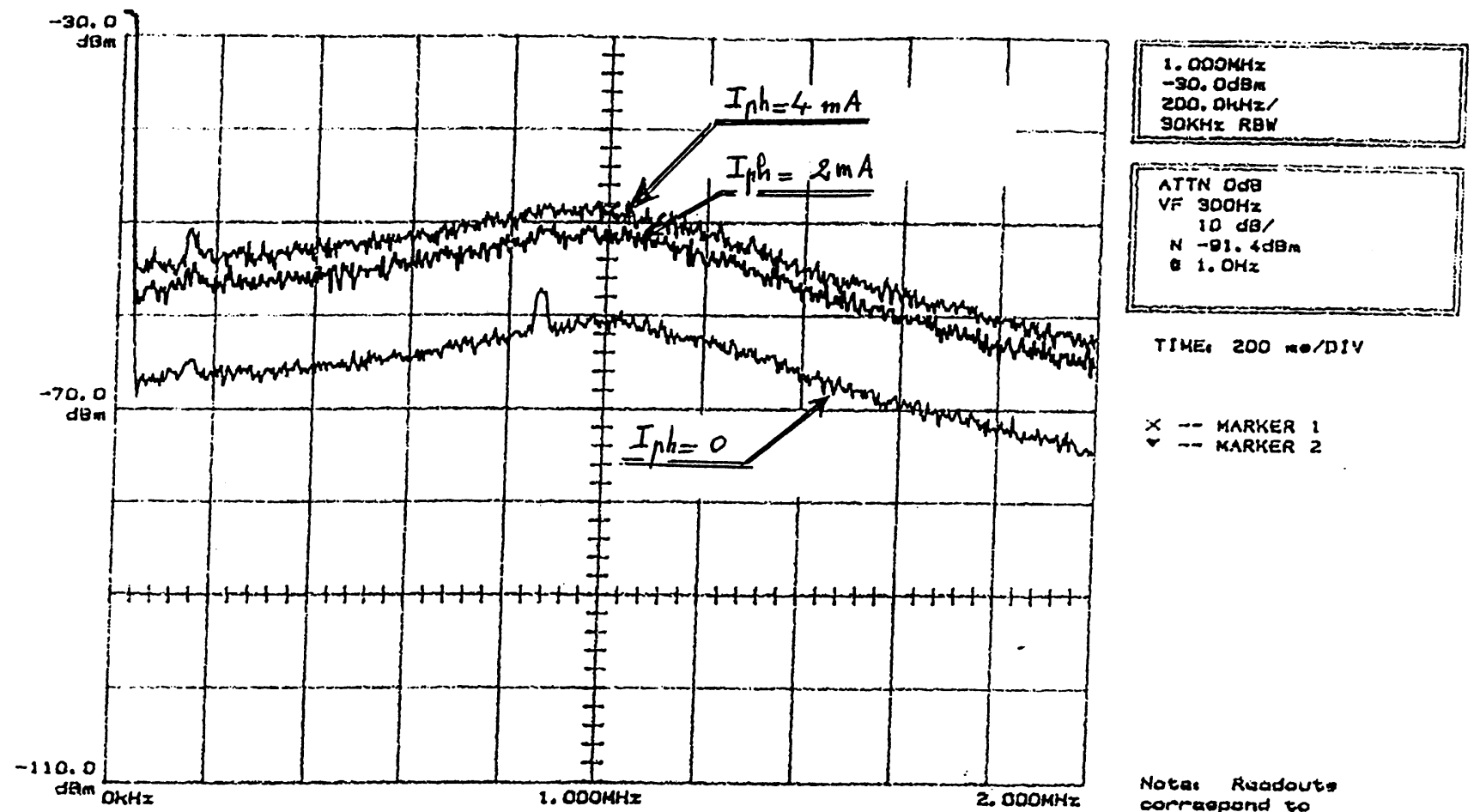

fig. 7

Noise for different mean currents in the photodiode $\left(I_{p h}=0,2\right.$ and $\left.4 \mathrm{~mA}\right)$

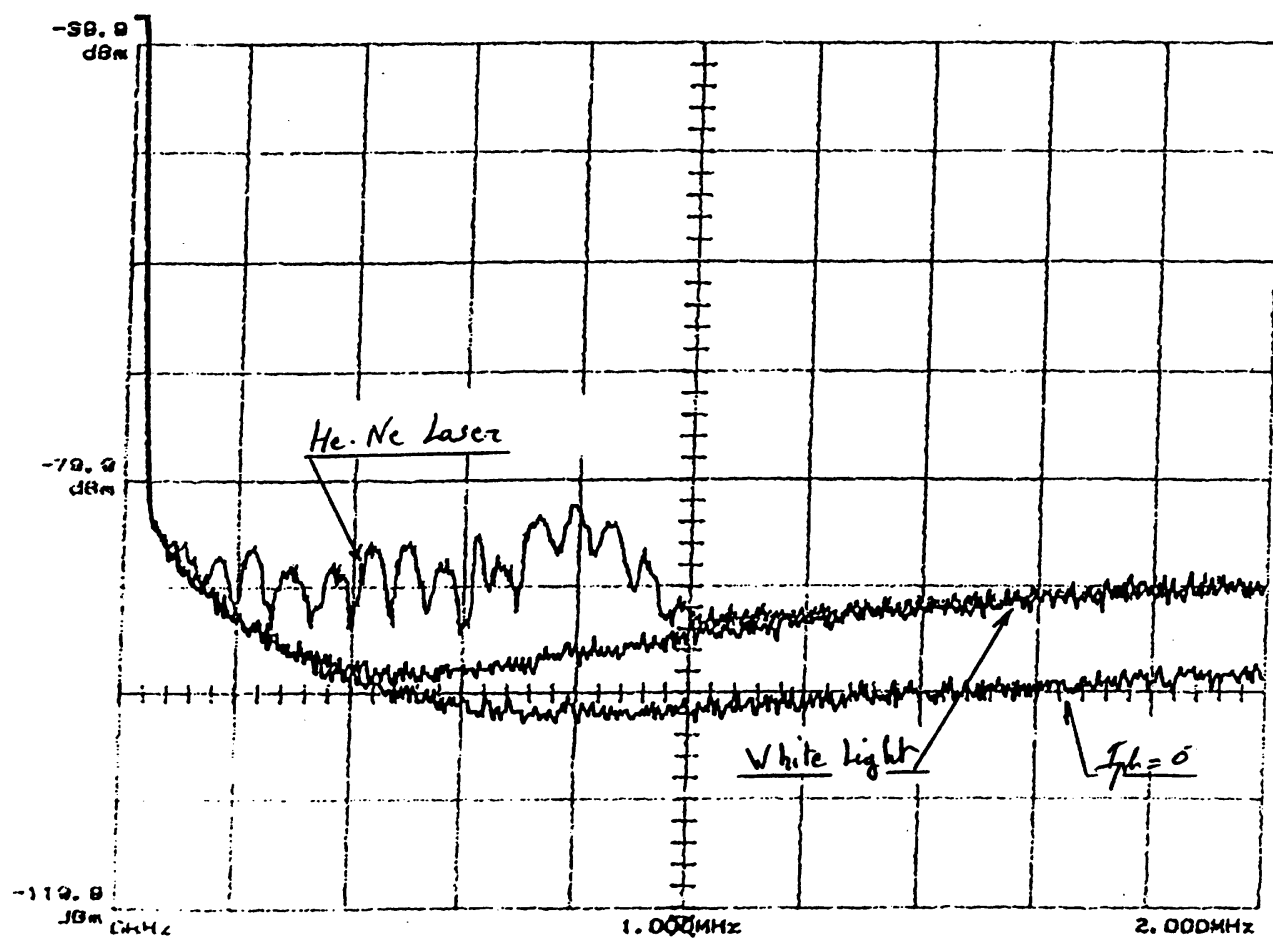

Notar Risadouts

corrapound to

waraform ' $\mathrm{C}$ '
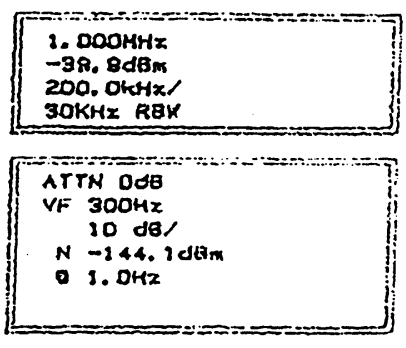

TIKES 200 mielOSV

× MARKER I

$$
I_{p}=\operatorname{lmA}
$$

fig 8

Noise comparison between $\mathrm{He}-\mathrm{Ne}$ laser $(5 \mathrm{~mW})$ and white light

Notar Rugdonte compampend to wovaform ' 0 " 


\section{4- Photon noise reduction}

\subsection{Photon noise reduction experiment:}

This last part presents a way to measure a photocurrent with noise power below the shot-noise limit. This experiment will prove that the photodetector is not responsible for the shot-noise. Shot-noise is related to the quantum « nature » of light and the shot-noise limit is due to the Poissonian statistics of the photons collected. This experiment will shaw that a suitable light source can give a sub-Poissonian statistics of photons collected and consequently a reduction of noise.

The idea is to use, as a light source, a high quantum efficiency light-emitting diode (Hamamatsu L2656 whose quantum efficiency is about 0.2 photons per electron at center wavelenght of $890 \mathrm{~nm}$ ). We put this LED as near as possible to the same photodiode (E.G.G. C30809) to collect as much photons as we can (we took off the window of the photodiode, and both led and photodiode are in a metal box).

Figure 9 represents the circuit which drives the LED either with a very low noise current produced by an usual stabilized power supply or with a « Poissonian " current produced by three photodiodes illuminated by any ordinary white light source. Changing the light level will change the mean current through the LED. It is easy to adjust the stabilized power supply and the light level in a way to get exactly the same mean current in the LED. This current, $I_{L E D}$, is measured by a voltmeter in parallel with the $100 \Omega$ resistance.

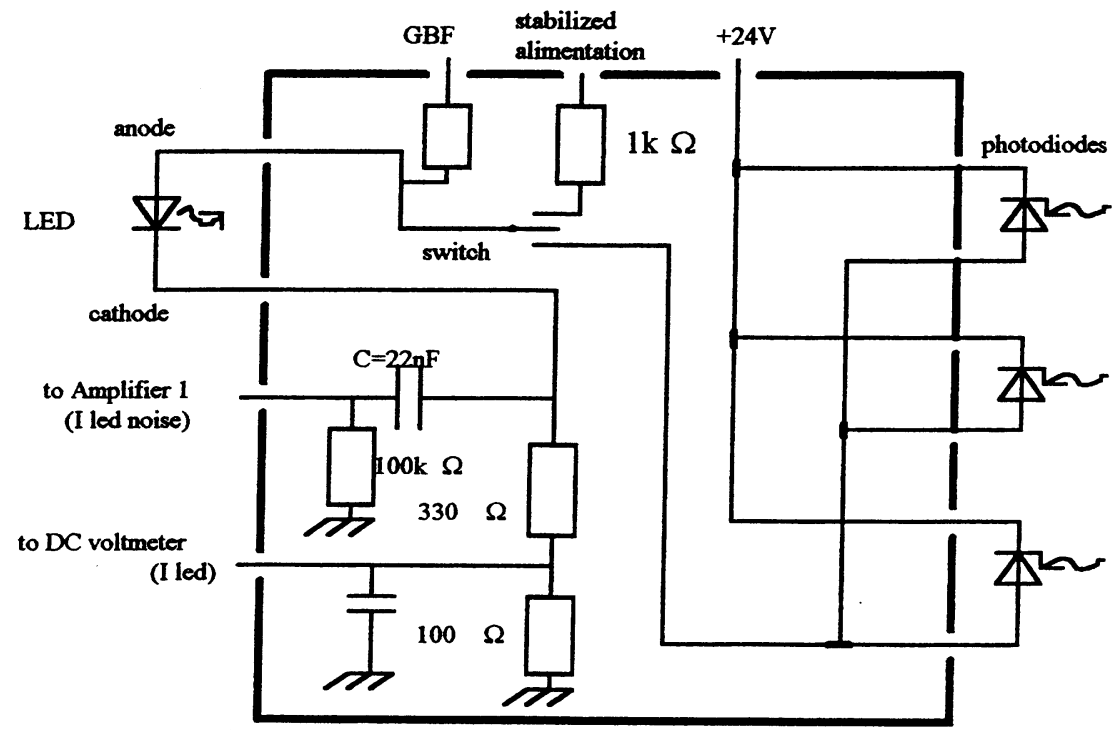

Fig. 9 : Setup of the sub-Poissonian/Poissonian light emitting device

Figure 10 explains clearly the principle of the shot-noise reduction experiment:

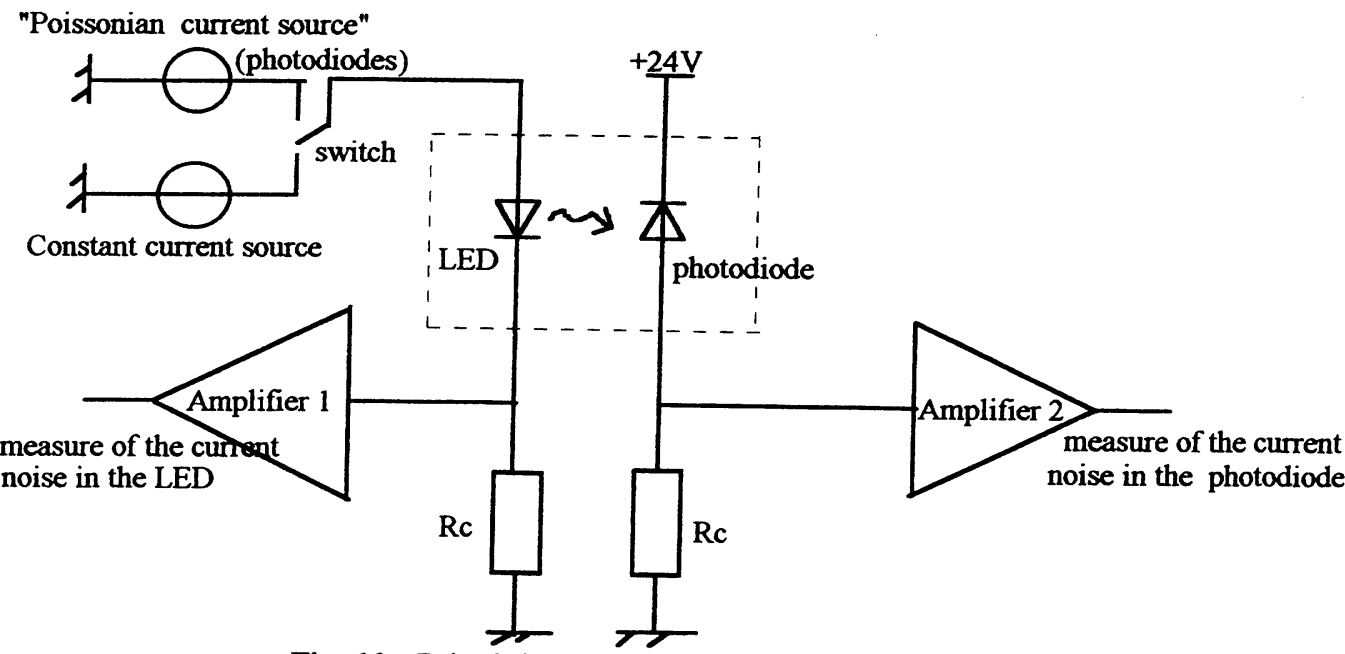

Fig. 10 : Principle of the shot-noise reduction experiment 
The amplifier 1 is the amplifier we called « thermal noise » in the first part of this paper (fig. 1). It allows the comparison of the noise on the current through the LED for both positions of the switch. Figure 11 shows noise power for each case with exactly the same mean current in the LED.

Noise power for the constant current source is about $20 \mathrm{~dB}$ below the noise power for the « Poissonian current source».

The amplifier 2 is the amplifier we called « photon noise " (fig. 5). We measure the amplifier voltage noise output with the spectrum analyser exactly in the same way we did for studying photon noise in part 3 of this paper. When the switch is in the position « Poissonian current source», we measure exactly the same noise as in part 3 with the same mean current in the photodiode. This « Poissonian current source " gives a calibration of the shot-noise level.

The experiment of «photon noise reduction " consists of verifying that the noise is below the shotnoise limit when the LED is driven by a constant current source. Figure 12 shows the noise reduction obtained for a mean current of $2 \mathrm{~mA}$ in the photodiode between $200 \mathrm{kHz}$ and $600 \mathrm{kHz}$. This reduction is $0.6 d B \pm 0.2$. The reduction becomes smaller above $600 \mathrm{kHz}$ which is the cut-off frequency of the LED.

The theoretical noise reduction is given by : $\Delta P(d B)=10 \log \left(1-\eta_{T}\right)$ where $\eta_{T}$ is the total quantum-efficiency for conversion from an electron through the LED to electron through the photodiode.

We measured : $\eta_{T}=\frac{I_{\text {photodiode }}}{I_{L E D}}=0.17$ which leads to a noise reduction theoretical value of $0.8 \mathrm{~dB}$.

\subsection{Simple explanation of the noise reduction:}

When a constant current source is used to drive the LED, the noise on the current is very low. The fluctuation of the number of electrons crossing the LED is very low in comparison to a " Poissonian statistics». If the quantum-efficiency of the LED was equal to one, the fluctuation of the number of photons emitted by the LED and collected by the photodiode would be very low too. The resulting noise reduction would be very large.

Unfortunately, the total quantum efficiency, $\eta_{T}$, is only 0.17 . This means that for six electrons crossing the LED only one electron on an average will cross the photodiode.

If we suppose the current in the LED is noiseless, the number of electrons through the L.E.D, $N_{e, L E D}$, during a time $\tau$ is constant. The number of electrons crossing the photodiode during a time $\tau$ is given by binomial distribution. The mean value and the variance of the number of electrons crossing the photodiode during a time $\tau$ are:

and :

$$
\left\langle n_{e, \text { photodiode }}\right\rangle=\eta_{T} N_{e, L E D}
$$

$$
\left\langle n_{e, \text { madodate }}^{2}\right\rangle=\eta_{T}\left(1-\eta_{T}\right) N_{e, L E D}
$$

This leads to :

and :

$$
I_{p h}=\left\langle N_{e, \text { photodiode }}\right\rangle \frac{e}{\tau}=\eta_{T} N_{e, L E D} \frac{e}{\tau}=\eta_{T} I_{L E D}
$$

$$
\begin{aligned}
& \left\langle\Delta i_{p h}{ }^{2}\right\rangle=\left\langle n_{\text {e.phocododock }}\right\rangle \frac{e^{2}}{\tau^{2}}=\eta_{T}\left(1-\eta_{T}\right) N_{\text {e.LBD }} \frac{e^{2}}{\tau^{2}} \\
& \left\langle\Delta i_{p h}{ }^{2}\right\rangle=\left(1-\eta_{T}\right) I_{\text {photodiode }} \frac{e}{\tau}=2 e I_{\text {photodiode }}\left(1-\eta_{T}\right) \Delta f
\end{aligned}
$$

So when the LED is driven by a constant current source, we find the variance of the fluctuation of the current in the photodiode is :

$$
\left\langle\Delta i_{p h}{ }^{2}\right\rangle=\left(1-\eta_{T}\right)\left\langle\Delta i_{p h}{ }^{2}\right\rangle_{\text {sho-noise }}
$$

The noise power is then multiplied by the factor : $F=1-\eta_{T}$ (called Fano factor) and $\Delta P(d B)=10 \log (F)$. $F$ is 0.83 in our experiment which leads to above mentionned $-0.8 \mathrm{~dB}$. 


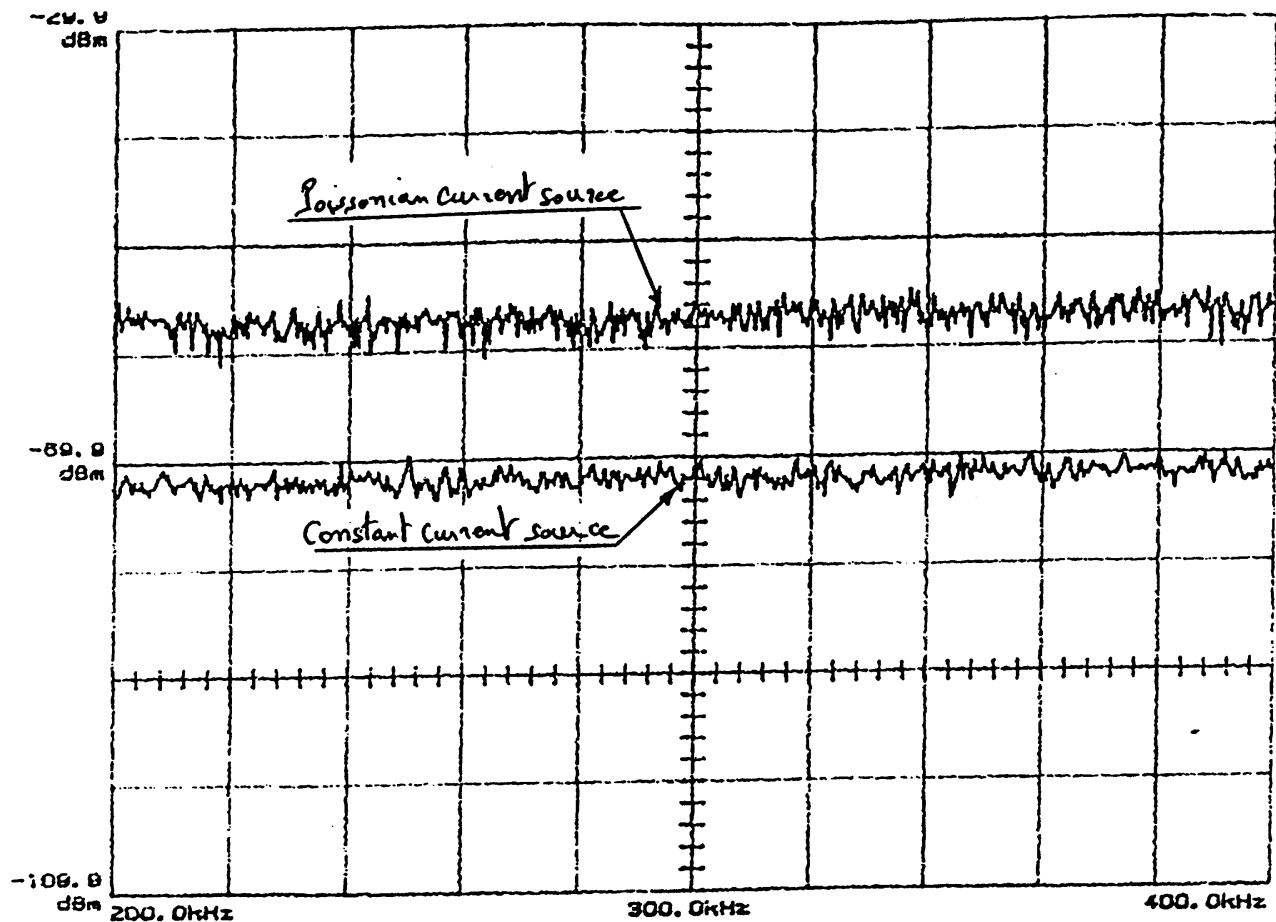

fig 11

Noise power in the LED for $I_{L E D}=15 \mathrm{~mA}$

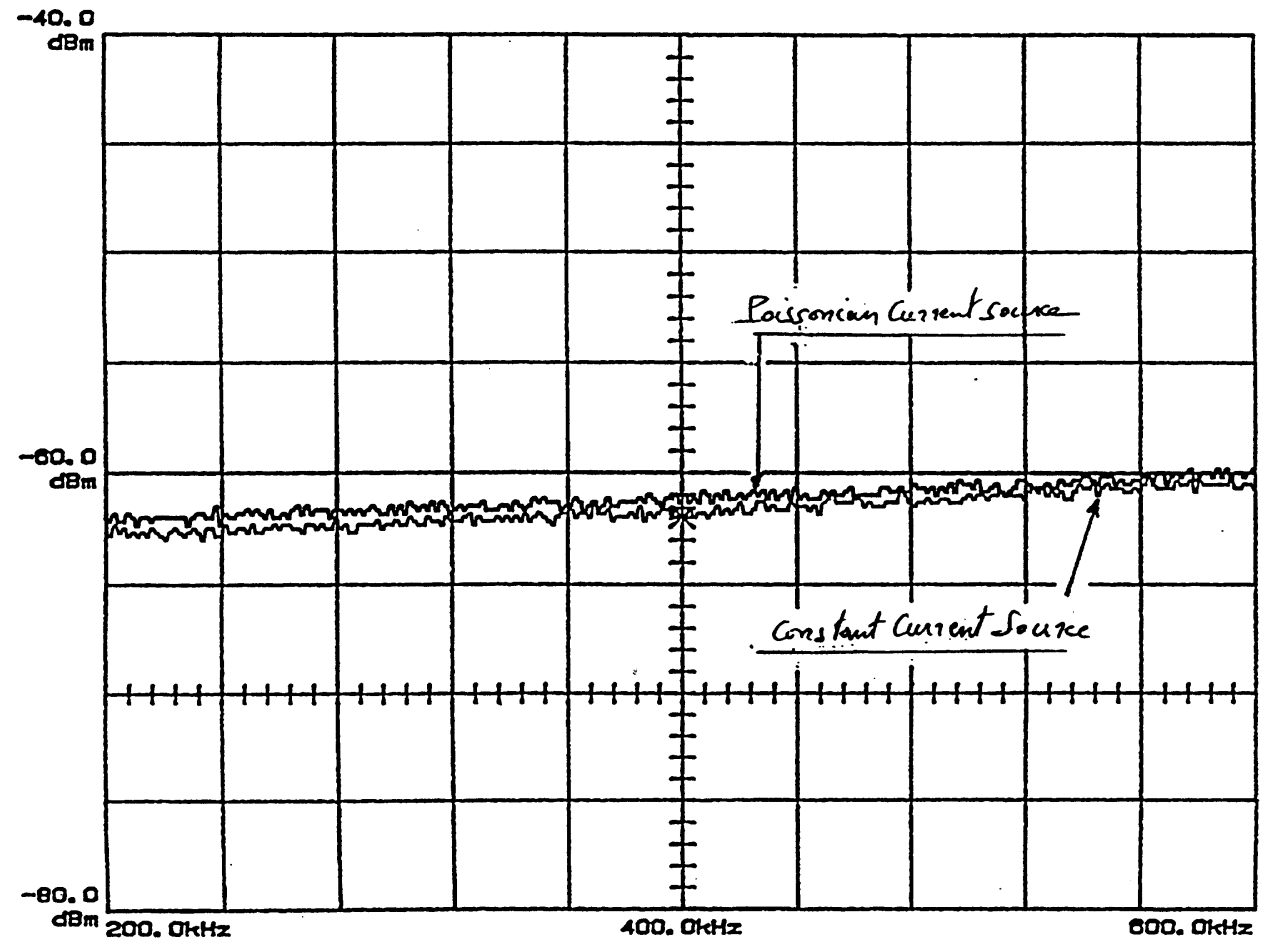

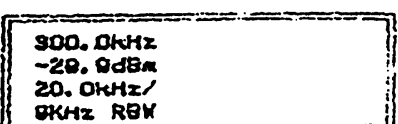

ATTK $3 d \theta$

VF $200 \mathrm{~Hz}$

10 ats

itutar Roovonte

compapand to

wovetarm ' $C$.

TIMES 200 AE/DIV

current noise

in the LED

$I_{\text {ted }}=11,76 \mathrm{~mA}$

400. OKHz
-40. OdBm
40. OKHz/
OKHz RBW

\begin{tabular}{|c|}
\hline $\begin{array}{l}\text { ATTN OdB } \\
\text { VF } 100 \mathrm{dI} \\
5 \mathrm{~dB} / \\
\mathrm{N}-98.0 \mathrm{dBm} \\
\text { a } 1.0 \mathrm{~Hz} \\
\text { AVE } 5\end{array}$ \\
\hline
\end{tabular}

TIMEI, 1 - TDIV

ENS AVE MODE. MEAN

$X$ - MARKER 2

- MARKER

PEAK MODE

Notar Readoute

fig 12

Photon noise reduction for $I_{\text {phorodiode }}=2 \mathrm{~mA}$ 


\section{5- Conclusion}

We described few experiments on noise sources in a photodetection system. A good photodetection system should be if possible shot-noise limited. We hope these experiments will help our students to understand and identify the different usual sources of noise. Our students should know how to measure noise, and signal to noise ratio. They have to understand the concepts of noise equivalent power and detectivity.

The last part, shot-noise reduction, is a way to go further in understanding that shot-noise is due to photon. Shot-noise reduction or squeezing is a purely quantum mechanical effect. It can not be described by a classical theory.

\section{6- Acknowledgments}

We wish to thank very sincerely Thierry Avignon for making all the electronic circuits, and Herve Sauer, Nathalie and Chriss Westbrook for helpfull discussions about this work.

\section{7-References}

Sub-Shot-Noise Manipulation of Light Using Semiconductor Emitters and Receivers J.-F. Roch,J.-Ph. Poizat and P. Grangier

Physical Review Letters Vol 71,Number 13 (1993)

Correlation Effects in Light Sources with High Quantum Efficiency

H.-A. Bachor, P. Rottengatter, and C.M. Savage

Appl. Phys. B 55, 258 (1992)

Semiconductor put the squeeze on light

$\mathrm{J}$ Ryan and M. Fox

Physics World Mars 1996 page 40

W.H. Richardson, S. Machida and Y. Yamamoto

Physical Review Letters Vol 66, 2867 (1991)

Photon Number Squeezed States in Semiconductor Lazers

Y. Yamamoto, W.H. Richardson, S. Machida

Science Vol. 255 p 1219 

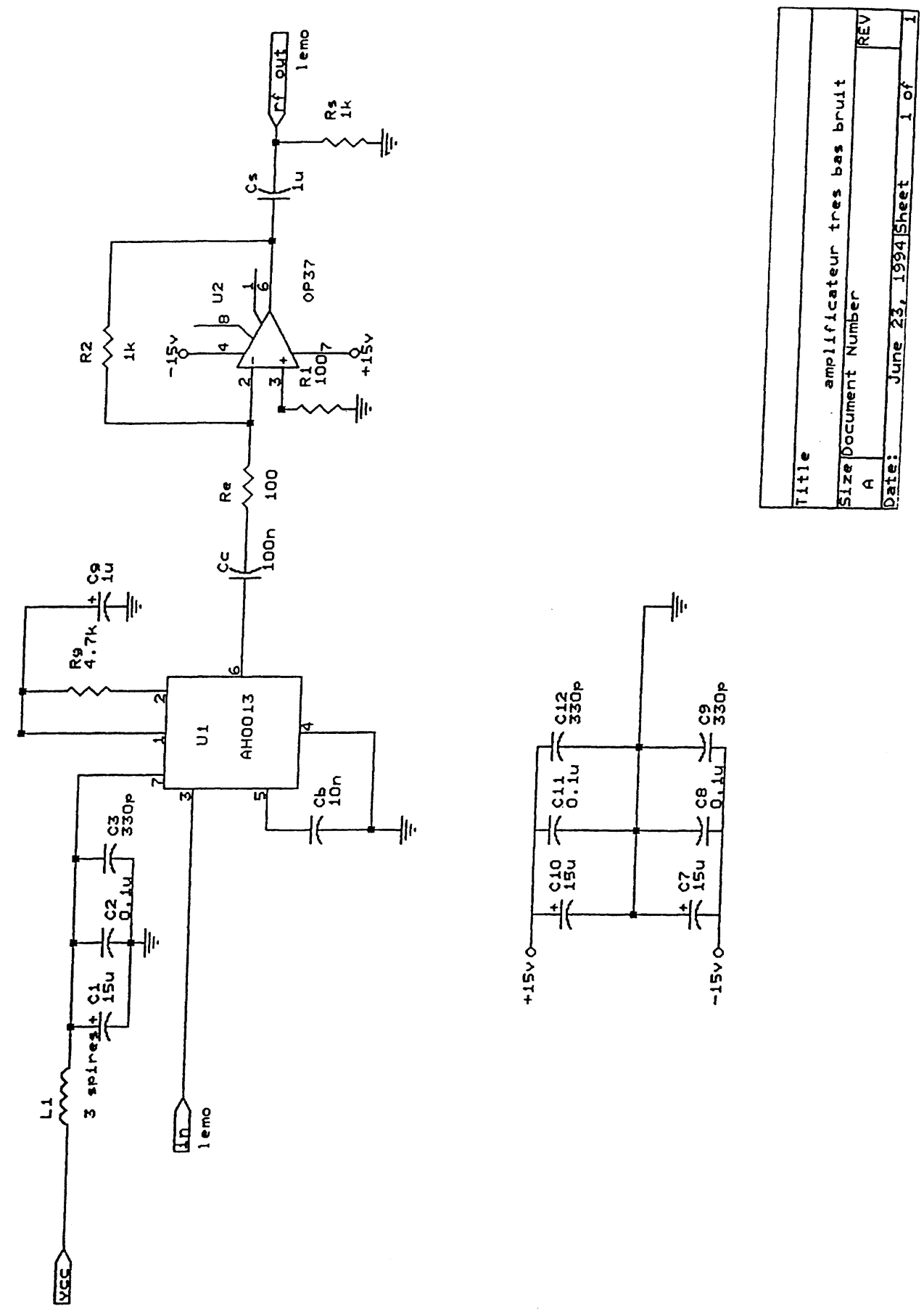

Amplifier 1 : Thermal noise 

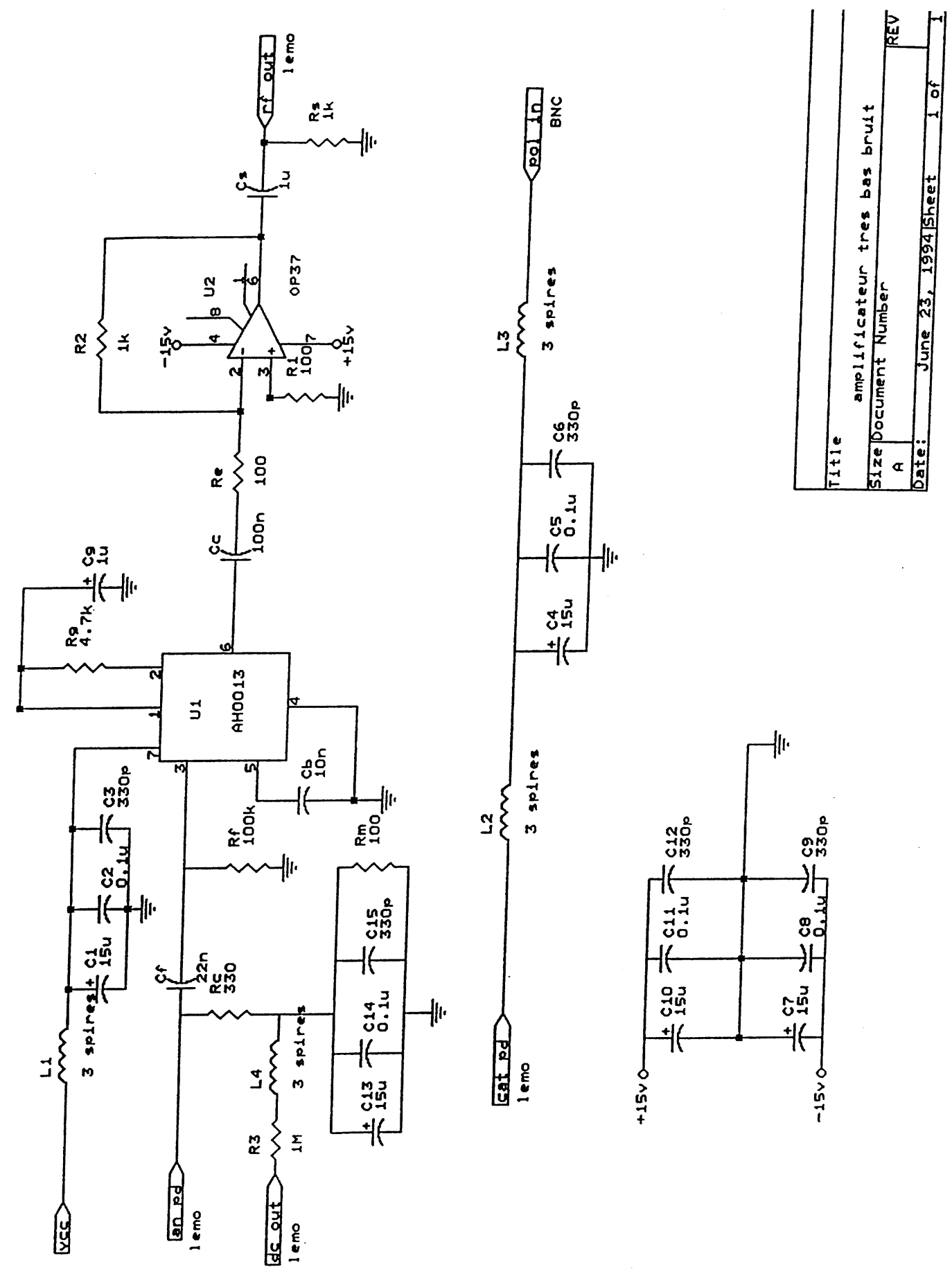

Amplifier 2 : Photon noise

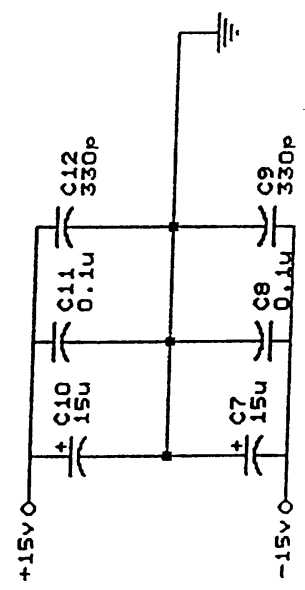

